\title{
EHMTI-0187. A novel ATP1A2 mutation in a case of familial hemiplegic migraine with especially severe attacks
}

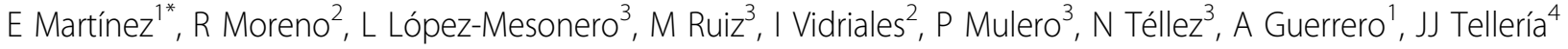 \\ From 4th European Headache and Migraine Trust International Congress: EHMTIC 2014 \\ Copenhagen, Denmark. 18-21 September 2014
}

\section{Introduction}

Familial hemiplegic migraine (FHM) is a rare disorder characterized by migraine attacks with motor weakness during aura phase. Mutations in CACNA1A, ATP1A2, SCN1A and PRRT2 genes have been described.

\section{Aim}

To describe a novel mutation in ATP1A2 gene in a FHM case with especially severe and prolonged symptoms.

\section{Patient}

22-year-old woman admitted due to migrainous headache and sudden onset right-side weakness and aphasia. Previous episodes during childhood. Her mother was diagnosed as hemiplegic migraine without genetic confirmation. Ten days before admission mild head trauma. On clinical exam, body temperature of $38^{\circ} \mathrm{C}$, diminished consciousness, left gaze preference, mixed aphasia, right facial palsy, right hemiplegia and left crural paresis. Urgent perfusion computed tomography showed hypoperfusion throughout all left cerebral hemisphere. No abnormalities in brain Magnetic Resonance and cerebrospinal fluid. In electroencephalogram diffuse slowing. Impaired consciousness and dysphasia began to improve three days after admission and mild dysphasia and right hemiparesis remained during 10 days. No recurrence during a six months follow-up.

\section{Results}

Genomic DNA was extracted from peripheral blood and exons were amplified using PCR primers. Capture and massive sequencing of exons of candidate genes obtained using SureSelect Human All Exon $51 \mathrm{Mb}$ kit (Agilent) and Hiseq2000 (Illumina) 30x tests. We identified an undescribed missense variant in heterozygous state in ATP1A2 gene (p.Thr364Met), pathogenic according to different prediction algorithms (SIFT, PolyPhen2, MutationTaster and Condel).

\section{Conclusion}

Genotype-phenotype correlation in FHM caused by ATP1A2 mutations is not well defined. This new mutation might be linked to especially severe and long lasting attacks.

No conflict of interest.

\section{Authors' details}

${ }^{1}$ Neurologist, Hospital Clínico Universitario de Valladolid, Valladolid, Spain. ${ }^{2}$ Clinical Analysis, Hospital Clínico Universitario de Valladolid, Valladolid, Spain. ${ }^{3}$ Neurology, Hospital Clínico Universitario de Valladolid, Valladolid, Spain. ${ }^{4}$ Genetics, Hospital Clínico Universitario de Valladolid, Valladolid, Spain.

Published: 18 September 2014

doi:10.1186/1129-2377-15-S1-B12

Cite this article as: Martínez et al:: EHMTI-0187. A novel ATP1A2 mutation in a case of familial hemiplegic migraine with especially severe attacks. The Journal of Headache and Pain 2014 15(Suppl 1):B12. 